Pacific Journal of Mathematics

CAUCHY TRANSFORMS AND CHARACTERISTIC FUNCTIONS 


\title{
CAUCHY TRANSFORMS AND CHARACTERISTIC FUNCTIONS
}

\author{
Kenneth C. Pietz
}

The following problem arises in the study of rational approximation: classify all plane sets $E$ such that $\hat{\mu}(z) \equiv$ $\int d \mu(\zeta) /(\zeta-z)=\chi_{E}(z)$ area almost everywhere for some complex Borel measure $\mu$. A partial solution to this problem for compact sets is given here. The main result is the following.

THEOREM. Let $K$ be a compact plane set with connected dense interior. Then there is a measure $\mu$ such that $\hat{\mu}=\chi_{K}$ area a.e., if and only if $K$ has finite Painlevé length.

1. Introduction. Throughout this paper, the word "measure" will mean a complex Borel measure supported on the complex plane C.. If $\mu$ is a compactly supported measure, we define the Newtonian potential of $\mu$ by the formula

$$
U_{|\mu|}(z)=\int \frac{d|\mu|(\zeta)}{|\zeta-z|}
$$

It is well known that $U_{|\mu|}$ is finite $d x d y$ a.e. For each $z$ such that $U_{|\mu|}(z)<\infty$ we define the Cauchy transform of $\mu$ by

$$
\hat{\mu}(z)=\int \frac{d \mu(\zeta)}{\zeta-z}
$$

The Cauchy transform is thus defined almost everywhere. We seek compact sets $K$ such that $\chi_{K}=\hat{\mu} d x d y$ a.e., for some $\mu$.

It is easy to see that we may assume that $K$ is connected. For, let $K=K_{1} \cup K_{2}$ with $K_{1}$ and $K_{2}$ closed and disjoint. Let $\hat{\mu}=\chi_{K}$ a.e., write $\mu_{i}$ for $\left.\mu\right|_{K_{i}}, i=1,2$ and define a function

$$
f=\left\{\begin{array}{ccc}
\hat{\mu}_{1} & \text { on } & \mathbf{C}-K_{1} \\
-\hat{\mu}_{2} & \text { on } & \mathbf{C}-K_{2}
\end{array}\right.
$$

By Liouville's theorem, $f \equiv 0$. It follows easily that $\hat{\mu}_{1}=\chi_{K_{1}}$ and $\hat{\mu}_{2}=\chi_{K_{2}}$. 
For a compact $K \subseteq \mathrm{C}$ we denote by $R(K)$ the Banach algebra of continuous functions on $K$ which are uniform limits of rational functions with poles off $K$. It is well known ([4]) that $\hat{\mu}=0$ on $\mathbf{C}-K$ if and only if $\mu \in R(K)^{\perp}$.

2. Painlevé Length. By a regular neighborhood of a compact plane set $K$ we mean an open set $V \supseteq K$ such that $\partial V$ consists of finitely many rectifiable curves surrounding $K$ in the usual sense of contour integration. We say that $K$ has finite Painleve length if there is a number $l$ such that every open $U \supseteq K$ contains a regular neighborhood $V$ of $K$ such that $\partial V$ has length at most $l$. The infimum of such numbers $l$ is called the Painlevé length of $K$.

The following theorem is well known, but we include a proof for completeness.

2.1. THEOREM. Let $K$ be a compact connected plane set with Painlevé length $\kappa<\infty$. Then there is a measure $\mu$ such that $\hat{\mu}=\chi_{K} d x d y$ a.e.

Proof. Let $\left\{U_{n}\right\}$ be a decreasing sequence of open sets such that

(i) $K=\bigcap_{n=1}^{\infty} U_{n}$

(ii) $\partial U_{j}$ is a rectifiable curve for each $j$

(iii) Length $\partial U_{j}<\kappa+\frac{1}{j}$.

Define $\mu_{j}=1 / 2 \pi i d z$ on $\partial U_{j}$ for each $j$. The sequence $\left\{\mu_{n}\right\}$ is bounded and hence a subsequence, again labeled $\left\{\mu_{n}\right\}$, converges weak-star to a limit $\mu$.

For any $\phi \in C_{0}^{\infty}$, we have

$$
\begin{aligned}
& \frac{1}{\pi} \iint \frac{\partial \phi}{\partial \bar{z}} \hat{\mu}(z) d x d y \\
& \quad=\int\left(-\frac{1}{\pi} \iint \frac{\partial \phi}{\partial \bar{z}} \frac{1}{z-\zeta} d x d y\right) d \mu(\zeta) \\
& \quad=\int \phi(\zeta) d \mu(\zeta)=\lim _{n} \int \phi(\zeta) d \mu_{n}(\zeta) \\
& \quad=\lim _{n} \frac{1}{\pi} \iint_{U_{n}} \frac{\partial \phi}{\partial \bar{z}} d x d y \\
& \quad=\frac{1}{\pi} \iint_{K} \frac{\partial \phi}{\partial \bar{z}} d x d y
\end{aligned}
$$

using the theorems of Green and Fubini. It follows easily that $\hat{\mu}=\chi_{K}$ a.e. 
The converse of this theorem is not true. This is easily seen by taking a closed disc, for example, and attaching a set with zero area but infinite Painlevé length. The converse can also fail when $K=\overline{K^{0}}$, as the following example shows.

2.2. EXAMPLE. Let $\left\{x_{i}\right\}_{i=1}^{\infty}$ be an enumeration of the rationals in $(0,1)$, let $\left\{r_{i}\right\}_{i=1}^{\infty}$ be any monotone decreasing sequence of positive numbers such that $\sum_{i=0}^{\infty} r_{i}<\infty$, and let $K_{0}=\{(x, y): x \in(0,1)$, $y=x \sin 1 / x\} \cup(0,0)$. We note that $K_{0}$ has infinite length.

Let $K=K_{0} \cup \cup_{n=1}^{\infty} \bar{\Delta}\left(P_{n} ; r_{n}\right)$, where $P_{n}=\left(x_{n}, x_{n} \sin 1 / x_{n}\right)$ and the $x_{n}$, $r_{n}$ are chosen inductively so that

(i) $\bar{\Delta}\left(P_{i} ; r_{i} \cap \bar{\Delta}\left(P_{j} ; r_{i}\right)=\phi\right.$ for $i \neq j$

(ii) $K_{0} \cap \bar{\Delta}\left(P_{j} ; r_{j}\right)$ is connected for each $j$

(iii) $\left\{x \in R:\left(x, x \sin \frac{1}{x}\right) \in K_{0}\right\}-\cup_{n=1}^{\infty} \bar{\Delta}\left(P_{n} ; r_{n}\right)$ contains no interval.

Evidently $K=\overline{K^{0}}$ and $K$ has infinite Painlevé length. But if we let $\mu=1 / 2 \pi i d z$ on the boundaries of the $\Delta\left(P_{n} ; r_{n}\right)$, we have $\hat{\mu}=\chi_{K}$ a.e.

The interior of the compact set in this example is dense, but not connected. In the next section we show that if $K^{0}$ is connected and dense in $K$, and if there is a measure $\mu$ such that $\hat{\mu}=\chi_{K}$ a.e., then $K$ must have finite Painlevé length.

3. Wermer's theorem and some extensions. The following theorem of John Wermer appears as a solution to a problem in [7].

THEOREM. Let $U$ be the region bounded by a Jordan curve $\Gamma$ and assume there is a measure $\mu$ on $\Gamma$ such that $\hat{\mu}(z)=1$ for $z \in U, \hat{\mu}(z)=0$ for $z \notin \Gamma \cup U$. Then $\Gamma$ is rectifiable.

We obtain some more general results, using ideas from Ahern and Sarason ([1]), Davie ([2]), and Gamelin and Garnett ([5]). However, many of the points in Wermer's original proof are retained.

The algebra $R(K)$ is called a Dirichlet algebra if it has no nonzero real annihilating measures.

Two points $p_{1}$ and $p_{2}$ of $K$ are said to be in the same Gleason part, or simply part, of $K$ if whenever $\left\{f_{n}\right\}$ is a sequence in $R(K)$ such that $\left\|f_{n}\right\|_{K} \leqq 1$ and $\left|f_{n}\left(p_{1}\right)\right| \rightarrow 1$, then also $\left|f_{n}\left(p_{2}\right)\right| \rightarrow 1$. This is an equivalence relation on $K$. 
A discussion of the properties of Dirichlet algebras and parts may be found in [4].

3.1 THEOREM. Let $K$ be a compact plane set such that $R(K)$ is a Dirichlet algebra. Assume $\mu$ is a measure such that $\hat{\mu}=1$ on $K^{0}, \hat{\mu}=0$ off $K$. Then the components $\left\{U_{i}\right\}_{i \in I}$ of $K^{0}$ are simply connected, $\partial U_{i}$ is a rectifiable curve for each $i$, and $\Sigma_{i \in I}$ length $\partial U_{i}<\infty$. Furthermore $\mu=1 / 2 \pi i d \zeta$ on $\cup_{i \in I} \partial U_{i}$ with appropriate orientation.

Proof. Theorem 5.1 of [5] implies that the components $\left\{U_{i}\right\}_{i \in I}$ of $K^{0}$ are simply connected, and Theorem 11.1 of [5] shows that the nontrivial parts of $K$ are precisely the $U_{i}$. Glicksberg's decomposition theorem (VI 3.4 of [4]) then gives $\mu=\sum_{i \in I} \mu_{i}$ where $\mu_{i}$ is supported on $\bar{U}_{i}$ for each $i$. Theorem VI 3.3 of [4] implies that $\mu_{i} \in R\left(\bar{U}_{i}\right)^{\perp}$ for each $i$ and it follows that $\hat{\mu}_{i}=1$ on $U_{i}, \mu_{i}=0$ off $\bar{U}_{i}$. It is easy to see that $R\left(\bar{U}_{i}\right)$ is Dirichlet for each $i$.

We may therefore restrict our attention to one pair $\left(\mu_{i}, U_{i}\right)$, which we relabel $(\mu, U)$. It is well known that $\mu$ is absolutely continuous with respect to harmonic measure for points in $U$, since $R(\bar{U})$ is Dirichlet.

By expanding $\hat{\mu}$ in a Laurent series, we obtain $\int_{\partial U} z^{k} d \mu(z)=$ $\delta_{-1, k}$. We can assume $0 \in U$. Let $\phi$ be the Riemann map of $\Delta=$ $\{|z|<1\}$ onto $U$ such that $\phi(0)=0$. Write $\rho_{0}$ for harmonic measure at 0 on $\partial \Delta$, and $\lambda_{0}$ the same on $\partial U$.

Lemma (Ahern-Sarason [1]; Davie [2]). The function $\phi$ has a measurable extension $\phi^{*}$ to a subset $E$ of $\partial \Delta$ of full measure such that $\phi^{*}$ is one-to-one on $E$ with a measurable inverse. The operator $T: L^{1}\left\{\lambda_{0}\right\} \rightarrow L^{1}\left\{\rho_{0}\right\}$ defined by $T f=f \circ \phi^{*}$ is an isometric isomorphism which maps $L^{\infty}\left\{\lambda_{0}\right\}$ isometrically onto $L^{\infty}\left\{\rho_{0}\right\}$.

Claim I. The function $1 / \phi^{*}$ is not in the $L^{\infty}\left\{\rho_{0}\right\}$ closure of the linear span of $\left\{\phi^{* k}: k \neq-1\right\}$. To see this, note that $\mu \ll \lambda_{0}$ implies $d \mu=g d \lambda_{0}$ for some $g \in L^{1}\left\{\lambda_{0}\right\}$ so that $T g \in L^{1}\left\{\rho_{0}\right\}$. Now suppose there is a sequence $\left\{Q_{j}\right\}_{j=1}^{\infty}$ of linear combinations of $\left\{\phi^{* k}: k \neq-1\right\}$ which converges to $1 / \phi^{*}$ in $L^{\infty}\left\{\rho_{0}\right\}$. Then also $Q_{j} T g \rightarrow 1 / \phi^{*} T g$ in $L^{1}\left\{\rho_{0}\right\}$ and $T^{-1}\left\{Q_{j}\right\} g \rightarrow z^{-1} g$ in $L^{1}\left\{\lambda_{0}\right\}$. But $\int T^{-1}\left\{Q_{j}\right\} g d \lambda_{0}=0$ for all $j$ and $\int z^{-1} g d \lambda_{0}=1$, a contradiction. This establishes the claim and shows that there is an $h \in L^{1}\left\{\rho_{0}\right\}$ such that $\int \phi^{* k} \bar{h} d \rho_{0}=\delta_{-1, k}$. 
Lemma (Ahern-Sarason [1]). Let $f \in H^{\infty}(U)$. Then there is a sequence $\left\{h_{n}\right\}_{n=1}^{\infty}$ in $R(\bar{U})$, with $\left\|h_{n}\right\|_{\infty} \leqq\|f\|_{\infty}$ for all $n$, such that $\left\{h_{n}(z)\right\} \rightarrow f(z)$ for all $z \in U_{0}$.

Claim II. The equality $\int \zeta \bar{h}(\zeta) d \rho_{0}(\zeta)=0$ holds. To prove this, apply the above lemma to $\phi^{-1}$. By Mergelyan's theorem ([4]), $R(\bar{U})$ is equal to $P(\bar{U})$, the uniform closure in $C(\bar{U})$ of the polynomials in $z$. Hence, there is a bounded sequence $P_{n}(z)$ of polynomials converging pointwise to $\phi^{-1}$ in $U$. So $\left\{P_{n}(\phi(\zeta))\right\} \rightarrow \zeta$ for all $\zeta \in \Delta$. By Alaoglu's theorem, there is a subsequence, again labeled $\left\{P_{n}\left(\phi^{*}\right)\right\}$ which converges weak-star on $\partial \Delta$ to some $\Psi$, i.e., converges over $L^{1}$. We need only show $\Psi=\zeta$. For fixed $k$,

$$
\frac{1}{2 \pi} \int_{0}^{2 \pi} \Psi\left(e^{i \theta}\right) e^{i k \theta} d \theta=\lim _{n \rightarrow \infty} \frac{1}{2 \pi} \int_{0}^{2 \pi} P_{n}\left(\phi^{*}\left(e^{i \theta}\right)\right) e^{i k \theta} d \theta=\delta_{-1, k} .
$$

So $\Psi$ and $\zeta$ have the same Fourier coefficients, and $\Psi=\zeta$. But now

$$
\begin{aligned}
0 & =\lim _{n \rightarrow \infty} \int_{\partial \Delta} P_{n}\left(\phi^{*}(\zeta)\right) \bar{h}(\zeta) d \rho_{0}(\zeta) \\
& =\int_{\partial \Delta} \zeta \bar{h}(\zeta) d \rho_{0}(\zeta) \\
& =\int_{\partial \Delta} \zeta \bar{h}(\zeta) d \rho_{0}(\zeta)
\end{aligned}
$$

which establishes the claim.

Similarly $\int \zeta^{k} \bar{h}(\zeta) d \rho_{0}(\zeta)=0$ for all $k \geqq 0$,and by the F. and M. Riesz theorem, $\bar{h} d \rho_{0}=w d z, w \in H^{1}$. Then for any $k, 0<r<1$,

$$
\int_{|z|=r} \phi^{k}(z) w(z) d z=\int_{|z|=1} \phi^{* k}(z) w(z) d z=\delta_{-1, k}
$$

But also $1 / 2 \pi i \int_{|z|=r} \phi^{k}(z) \phi^{\prime}(z) d z=\delta_{-1, k^{\prime}}$ so $\left(w(z)-\phi^{\prime}(z) / 2 \pi i\right) d z$ annihilates all integral powers of $\phi^{*}$, hence all integral powers of $z$, so that $w(z)=\phi^{\prime}(z) / 2 \pi i$, and $\phi^{\prime} \in H^{1}$. This implies that $\partial U$ is a rectifiable Jordan curve (see e.g., [3], p. 44). The theorem is now clear.

By similar methods we can prove:

3.2 THEOREM. Let $K$ be a compact plane set such that $\operatorname{Re}(R(K))$ has finite defect in $C_{R}(\partial K)$. Then the components $\left\{U_{i}\right\}_{i \in I}$ of $K^{\circ}$ are finitely connected and there is a measure $\mu$ on $\partial K$ such that $\hat{\mu}=1$ on $K^{\circ}$, $\hat{\mu}=0$ off $K$ if and only if the following three conditions hold. 
(i) For each i, $\partial U_{i}$ is a cycle composed of rectifiable curves.

(ii) $\sum_{i \in I}$ length $\partial U_{i}<\infty$

(iii) $\mu=\frac{1}{2 \pi i} d \zeta$ on $\cup_{i \in I} \partial U$ with appropriate orientation.

3.4 THEOREM. Let $K$ be a compact plane set with connected dense interior. Then there is a measure $\mu$ with $\hat{\mu}=1$ on $K^{0}, \hat{\mu}=0$ off $K$ if and only if

(i) The components of $\mathbf{C}-K$ are bounded by rectifiable curves $\left\{\gamma_{i}\right\}_{i \in I}$ with finite total length and

(ii) $\mu=1 / 2 \pi i d \zeta$ on $\cup_{i \in I} \gamma_{i}$ with appropriate orientation.

Proof. As before, the sufficiency of the two conditions is obvious. To prove the necessity, let $\Delta$ be a large disk containing $K$, and let $\lambda=1 /\left.2 \pi i d \zeta\right|_{\partial \Delta}-\mu$. Then $\hat{\lambda}=1$ on $\left(\bar{\Delta}-K^{0}\right)^{0}=\Delta-K$, and $\hat{\lambda}=0$ off $\bar{\Delta}=K^{0}$.

The hypotheses imply that $\bar{\Delta}-K^{0}$ is finitely connected. In fact, the complement of $\bar{\Delta}-K^{0}$ has two components, $\mathbf{C}-\bar{\Delta}$ and $K^{0}$. Also, the components of $\left(\bar{\Delta}-K^{0}\right)^{0}=\Delta-K$ are simply connected. As before, $R\left(\Delta-K^{0}\right)$ is a Dirichlet algebra so we can apply Theorem 3.1 to $\bar{\Delta}-K^{0}$. The conclusions (i) and (ii) follow easily.

3.4 CoROllary. Let $K$ be a compact plane set with connected dense interior. Then there is a measure $\mu$ with $\hat{\mu}=\chi_{\mathrm{K}} d x d y$ a.e. if and only if $K$ has finite Painlevé length.

\section{REFERENCES}

1. P. R. Ahern and Donald Sarason, On Some Hypo-Dirichlet Algebras of Analytic Functions, Amer. J. Math., 89, 4 (1967), 932-941.

2. A. M. Davie, Dirichlet Algebras of Analytic Functions, J. Functional Analysis, 6 (1970), 348-356.

3. P. Duren, Theory of $H^{p}$ spaces, Academic Press, New York, 1970.

4. T. W. Gamelin, Uniform Algebras, Prentice-Hall, Englewood Cliffs, N. J., 1969.

5. T. W. Gamelin and John Garnett, Pointwise Bounded Approximation and Dirichlet Algebras, J.

Functional Analysis, 8 (1971), 360-404.

6. John Garnett, Analytic capacity and Measure, Lecture Notes in Math. No. 297, Springer, 1972.

7. John Wermer, Solution to a Problem in Advanced Problems and Solutions, Amer. Math. Monthly, 64 (1957), 372.

Received June 10, 1974. This paper contains material from the author's thesis, submitted to the University of California, Los Angeles. The author would like to thank his advisor, John Garnett, for his help and encouragement.

LockHeEd Electronics Company, Houston, TeXas

AND

University of California, Los ANgeles, California 


\section{PACIFIC JOURNAL OF MATHEMATICS}

\section{EDITORS}

RICHARD ARENS (Managing Editor)

University of California

Los Angeles, California 90024

\section{J. DugundJI}

Department of Mathematics University of Southern California Los Angeles, California 90007

D. Gilbarg and J. Milgram

Stanford University

Stanford, California 94305

\section{ASSOCIATE EDITORS}
E. F. BECKENBACH
B. H. NeumanN
F. WoLF
K. YoshiDA

\section{SUPPORTING INSTITUTIONS}

\author{
UNIVERSITY OF BRITISH COLUMBIA \\ CALIFORNIA INSTITUTE OF TECHNOLOGY \\ UNIVERSITY OF CALIFORNIA \\ MONTANA STATE UNIVERSITY \\ UNIVERSITY OF NEVADA \\ NEW MEXICO STATE UNIVERSITY \\ OREGON STATE UNIVERSITY \\ UNIVERSITY OF OREGON \\ OSAKA UNIVERSITY
}

\author{
UNIVERSITY OF SOUTHERN CALIFORNIA \\ STANFORD UNIVERSITY \\ UNIVERSITY OF TOKYO \\ UNIVERSITY OF UTAH \\ WASHINGTON STATE UNIVERSITY \\ UNIVERSITY OF WASHINGTON \\ AMERICAN MATHEMATICAL SOCIETY
}

The Supporting Institutions listed above contribute to the cost of publication of this Journal, but they are not owners or publishers and have no responsibility for its contents or policies.

Mathematical papers intended for publication in the Pacific Journal of Mathematics should be in typed form or offset-reproduced (not dittoed), double spaced with large margins. Underline Greek letters in red, German in green, and script in blue. The first paragraph or two must be capable of being used separately as a synopsis of the entire paper. Items of the bibliography should not be cited there unless absolutely necessary, in which case they must be identified by author and Journal, rather than by item number. Manuscripts, in duplicate, may be sent to any one of the four editors. Please classify according to the scheme of Math. Reviews, Index to Vol. 39. All other communications should be addressed to the managing editor, or Elaine Barth, University of California, Los Angeles, California, 90024.

100 reprints are provided free for each article, only if page charges have been substantially paid. Additional copies may be obtained at cost in multiples of 50 .

The Pacific Journal of Mathematics is issued monthly as of January 1966. Regular subscription rate: $\$ 72.00$ a year (6 Vols., 12 issues). Special rate: $\$ 36.00$ a year to individual members of supporting institutions.

Subscriptions, orders for back numbers, and changes of address should be sent to Pacific Journal of Mathematics, 103 Highland Boulevard, Berkeley, California, 94708.

PUBLISHED BY PACIFIC JOURNAL OF MATHEMATICS, A NON-PROFIT CORPORATION Printed at Jerusalem Academic Press, POB 2390, Jerusalem, Israel.

$$
\begin{gathered}
\text { Copyright (C) } 1975 \text { Pacific Journal of Mathematics } \\
\text { All Rights Reserved }
\end{gathered}
$$




\section{Pacific Journal of Mathematics}

\section{Vol. 58, No. $2 \quad$ April, 1975}

Zvi Artstein and John Allen Burns, Integration of compact set-valued functions . . . . . . . . . 297

Mark Benard, Characters and Schur indices of the unitary reflection group $[321]^{3} \ldots \ldots \ldots . .309$

Simeon M. Berman, A new characterization of characteristic functions of absolutely continuous

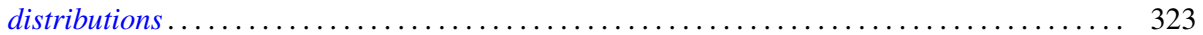

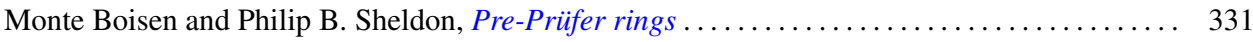

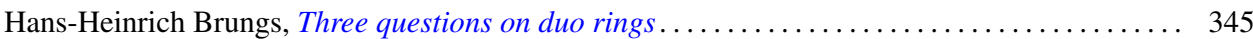

Iracema M. Bund, Birnbaum-Orlicz spaces of functions on groups................. 351

John D. Elwin and Donald R. Short, Branched immersions between 2-manifolds of higher

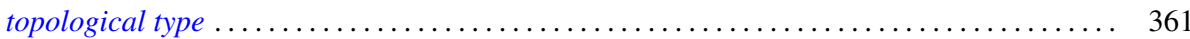

Eric Friedlander, Extension functions for rank 2, torsion free abelian groups . .......... 371

Jon Froemke and Robert Willis Quackenbush, The spectrum of an equational class of

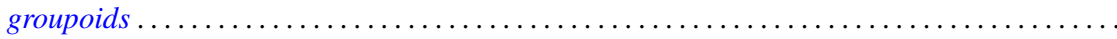

Barry J. Gardner, Radicals of supplementary semilattice sums of associative rings ...........

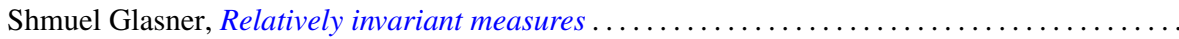

George Rudolph Gordh, Jr. and Sibe Mardesic, Characterizing local connectedness in inverse

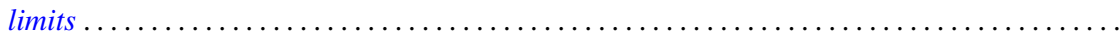

Siegfried Graf, On the existence of strong liftings in second countable topological spaces......

Stanley P. Gudder and D. Strawther, Orthogonally additive and orthogonally increasing

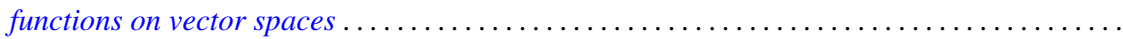

Darald Joe Hartfiel and Carlton James Maxson, A characterization of the maximal monoids and

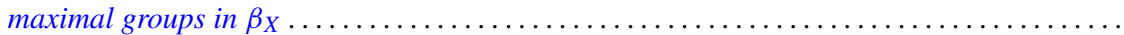

Robert E. Hartwig and S. Brent Morris, The universal flip matrix and the generalized faro-shuffle. .

William Emery Haver, Mappings between ANRs that are fine homotopy equivalences. .

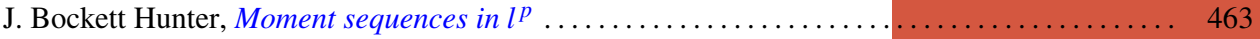

Barbara Jeffcott and William Thomas Spears, Semimodularity in the completion of a poset.... 467

Jerry Alan Johnson, A note on Banach spaces of Lipschitz functions . . . . . . . . . . . . 475

David W. Jonah and Bertram Manuel Schreiber, Transitive affine transformations on

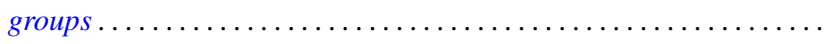

Karsten Juul, Some three-point subset properties connected with Menger's characterization of

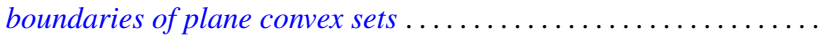

Ronald Brian Kirk, The Haar integral via non-standard analysis . . . . . . . . . . . . . 517

Justin Thomas Lloyd and William Smiley, On the group of permutations with countable

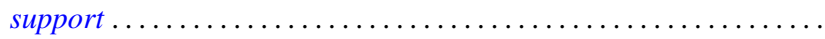

Erwin Lutwak, Dual mixed volumes .................................. 531

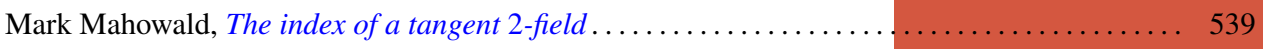

Keith Miller, Logarithmic convexity results for holomorphic semigroups . . . . . . . . . . . . 549

Paul Milnes, Extension of continuous functions on topological semigroups . . . . . . . . . . 553

Kenneth Clayton Pietz, Cauchy transforms and characteristic functions ................ 563

James Ted Rogers Jr., Whitney continua in the hyperspace $C(X) \ldots \ldots \ldots \ldots \ldots \ldots \ldots \ldots .569$

Jean-Marie G. Rolin, The inverse of a continuous additive functional . . . . . . . . . . . . 585

William Henry Ruckle, Absolutely divergent series and isomorphism of subspaces . ........ 605

Rolf Schneider, A measure of convexity for compact sets . ..................... 617

Alan Henry Schoenfeld, Continous measure-preserving maps onto Peano spaces .......... 627

V. Merriline Smith, Strongly superficial elements .......................... 643

Roger P. Ware, A note on quadratic forms over Pythagorean fields . . . . . . . . . . . . . . 651

Roger Allen Wiegand and Sylvia Wiegand, Finitely generated modules over Bezout rings . . . . 655

Martin Ziegler, A counterexample in the theory of definable automorphisms . . . . . . . . . 665 